\title{
Las asociaciones del Tercer Sector, un nuevo objeto para las ciencias de la gestión
}

\author{
Madeleine Richer*
}

\section{Resumen}

Las asociaciones sin fines de lucro o tercer sector están siendo objeto de un nuevo interés por parte de los investigadores. El objetivo de este artículo es presentar, a partir de una revisión de la literatura reciente sobre el tema, y de una investigación en curso sobre las asociaciones de la economía social en Canadá, algunos problemas para el estudio de este objeto. En primer lugar, el problema de la definición y clasificación de las asociaciones, en segundo lugar, las particularidades de la gestión asociativa. Se concluye que existen diferentes enfoques para la clasificación de las asociaciones y que el concepto de economía social no es utilizado en todos los países, se concluye también que la evolución reciente de las asociaciones se caracteriza por una creciente importancia de la gestión y por una mayor capacidad empresarial de estas organizaciones, sin embargo, existen diferencias fundamentales entre las asociaciones que conforman la economía social y las empresas privadas clásicas; la combinación de objetivos sociales y económicos hace la gestión más compleja.

Palabras claves: Asociaciones, economía social, tercer sector, gerencia.

\section{Third sector associations, a new area in administrative sciences}

\section{Abstract}

This paper, based on a review of recent litterature and an a research in course on the associations that from Canadian social economy, presents some of the principal issues that appear in the study of associations. The principal conclusions are that there are

Recibido: 29-01-97. Aceptado: 08-05-97

* Historiadora, Maestría en Intervensión Social. Investigadora del Centro de Estudios de la Empresa, FCES - LUZ. 
different criteria for the classification of associations, and in many countries the concept of social economy is no used. Also the recent changes observed in the monoprofit sector show an increased importance of management. But the management in these organisations has some particularities if compared with the management of classical private enterprises; the combination of social and economical objetives makes the management more complex.

Key words: Associations, social economy, third sector, management.

\section{Introducción}

Por diversas razones, las ciencias económicas y administrativas han ignorado durante mucho tiempo el mundo de las asociaciones $\sin$ fines de lucro. Sin embargo, juegan un rol importante en la vida económica y social de muchos países. En varios campos de actividad: salud, servicios sociales, educación, etc. las organizaciones sin fines de lucro del "tercer sector" o de la "economía social" están asumiendo un papel cada vez más importante en el contexto de la crisis del modelo de desarrollo.

Desde los años ochenta, en prácticamente todos los paises se observa una toma de conciencia de la importancia de las realidades económicas de un tercer tipo, distinto del sector público y del sector privado lucrativo. Esta toma de conciencia ha generado nuevas corrientes de investigación e importantes esfuerzos para comprender mejor el funcionamiento de las organizaciones del "tercer sectorn.

Se ha venido conformando el tercer sector como un nuevo campo de estudio que se institucionaliza en centros de investigación universitarios, en varios países. Se puede mencionar por ejemplo el grupo formado a mediados de los años ochenta en la Universidad de Yale y coordinado por Lester Salamon, el John Hop- kins Comparative Nonprofit Sector Project, que agrupa investigadores de unos 12 paises (Anheier y Seibel, 1990). Otro importante grupo internacional de investigación es el del Centro Interuniversitario de Investigación, Información y Enseñanza sobre las Cooperativas (CIRIEC), donde se creó en 1988 la Comisión Cientifica Internacional para la Economia Social. Se publicó en 1992 un primer libro con los trabajos de 20 investigadores sobre la economía social en 9 países (Monzon y Defourny, 1992).

El nuevo rol del tercer sector en el contexto de la crisis del modelo de desarrollo (crisis de la economia salarial y del Estado de bienestar) y las transformaciones ocurridas en las mismas asociaciones contribuyen también a explicar el nuevo interés de las ciencias sociales por este objeto. Estas transformaciones afec$\tan$ las fórmulas de financiamiento, los modos de acción, las relaciones con los poderes públicos y los modelos organizacionales de las asociaciones (Defourny, 1992)

En este artículo, discutimos algunos enfoques conceptuales sobre las asociaciones $\sin$ fines de lucro, el tercer sector y la economía social, y algunas especificidades de la gestión asociativa. 


\section{Tercer sector, organizaciones no lucrativas o economía social.}

Según los autores norteamericanos Salamon y Anheier (1992), la falta de atención prestada al sector no lucrativo hasta hace pocos años se debe a factores tanto conceptuales como empíricos. Pocos paises recolectan y procesan información sobre el tercer sector como categoría separada en el sistema de cuentas nacionales. $Y$ a la penuria de datos estadísticos se suman las diferencias en la conceptualización de las asociaciones. El término "tercer sector" tiene un carácter residual que le da cierta imprecisión. En algunos países, principalmente de tradición anglosajona, el tercer sector se define por las asociaciones sin fines de Iucro, llamadas nonprofit organizations en los Estados Unidos y voluntary organizations en el Reino Unido. Las primeras tienen como linea de demarcación la elegibilidad a un estatus especial tributario por parte de las autoridades fiscales (Rock y Klinedinst, 1992, 289). Las segundas no estan definidas por ley en el Reino Unido ni tampoco existe una estructura jurídica particular asociada a las organizaciones voluntarias. Pero el término sector voluntario, aúnque su cobertura exacta sea problemática, incluye para el sector público y la mayoría de los ciudadanos las organizaciones que presentan las siguientes condiciones: que estén formalmente constituídas, privadas, autogobernadas, sus fines principales no sean mercantiles, no distribuyan beneficios a aquellas personas que las controlan, se beneficien de algún trabajo volun- tario y que no estén ligadas a ningún partido político ni religión (Kendall et al., 1992; 91 ).

Las grandes lineas de investigación que atraviesan la literatura sobre las organizaciones sin fines de lucro (o.s.f.l.) se refieren, por una parte a la explicación de la existencia y del rol de estas organizaciones en las diferentes sociedades. Por ejemplo, los investigadores se preguntan porqué las o.s.f.I. son más importantes en ciertas sociedades que en otras; por que tienen más presencia en ciertas actividades. Otras investigaciones se interesan por el funcionamiento de las o.s.f.l., por sus semejanzas y diferencias con las empresas privadas o por su eficacia y eficiencia productiva (Anheier y Seibel, 1990).

En algunos paises, se ha venido construyendo el concepto de economía social, que incluye tres grandes tipos de organizaciones: las cooperativas, las sociedades mutualistas y las asociaciones $\sin$ fines de lucro que realizan aiguna actividad económica. Estas agrupaciones tienen en común su organización interna: el poder no depende en ellas de la importancia del capital invertido; y los beneficios que se realizan eventualmente no se reparten entre los miembros. El término "economia social" sin embargo no es utilizado en todos los países, y en muchas sociedades, las cooperativas y mutualidades no son conceptualizadas como parte de una misma categoría junto con las asociaciones sin fines de lucro: se considera fundamental la diferencia que existe entre las organizaciones que benefician sólo a sus miembros, como las cooperativas, y las organizaciones sin fi- 
nes de lucro que atienden a una población de no miembros, como por ejemplo, las organizaciones caritativas. En esta perspectiva, las cooperativas no forman parte del sector no lucrativo.

Un factor importante en la constitución de la economía social como objeto de estudio es el reconocimiento de un conjunto de organizaciones como pertenecientes a una misma categoria. Es un reconocimiento que tiene tres dimensiones: un reconocimiento mutuo de las organizaciones, que reconocen una identidad común entre ellas; un reconocimiento político, por los poderes públicos, y un reconocimiento por el medio científico (Malo, 1991).

El reconocimiento mutuo se manifiesta en la creación de agrupaciones de asociaciones. que en algunos paises reunen asociaciones pertenecientes a los más diversos sectores de actividad.

Un factor importante de este reconocimiento mutuo es el contexto de la crisis del modelo fordista y del Estado de bienestar, donde se redefinen las relaciones entre sector público y sector privado, en el marco de los procesos de descentralización y privatización, y de la formación de una ceconomía mixta de bienestar" (Kramer, 1990).

Por ejemplo en Francia se formó a mediados de los años setenta el Comité Nacional para el establecimiento de vínculos entre las cooperativas, las mutualidades y las asociaciones (CNLAMCA) que se constituyó en un importante factor de unidad de las asociaciones pertenecientes a la economía social. Podían pertenecer al CNLAMCA todas las asociaciones que respetaran los cuatro principios: libertad de adhesión, gestión democráti$\mathrm{ca}$, no lucratividad, o independencia respecto del Estado. EI CNLAMCA realizó un coloquio nacional para manifestar su existencia y su importancia: veinte millones de miembros y doscientos mil empleos. En 1980, el Protocolo de acuerdo que había constituido la primera manifestación pública de su reunión se convierte en la Carta de la Economía Social (Vienney, 1994, 4).

Si bien en Francia las asociaciones sin fines de lucro son consideradas como la tercera gran familia de la economía social, junto con las cooperativas y las mutualidades, en otros paises, como en España, no existen como fuerza organizada aúnque algunos sectores hayan empezado a agruparse en estructuras de un nivel superior. En Venezuela, en el periodo reciente, se han producido algunos agrupamientos de asociaciones, en forma de redes. Un ejemplo es la Coordinadora de ong que realiza trabajo con niños (Congani). La heterogeneidad de las actividades que realizan las asociaciones, de los grupos sociales que las constituyen asi como de las tradiciones ideológicas a las que pertenecen, hacia difícil hasta hace poco este proceso de agrupamiento. La crisis económica y la criris de los modelos ideológicos crean condiciones propicias a un acercamiento entre asociaciones.

El reconocimiento mutuo y el reconocimiento por el Estado constituyen un proceso interactivo. Por un lado, existen organizaciones que tienden a reconocerse entre si como constituyendo un mismo conjunto, aunque tengan estatus jurídicos, actividades económicas y composi- 
ciones sociales distintas. Por otro lado, se solidarizan para hacer reconocer sus caracteres comunes por los poderes públicos.

El reconocimiento público se puede manifestar en la legislación o en diversas reglamentaciones y normas. Por ejemplo, existe en muchos países una Ley de Cooperativas que define las condiciones de pertenencia a este tipo de organización. Las organizaciones sin fines de lucro pueden también estar definidas en una ley: por ejemplo, la Ley de 1901 en Francia, que define la asociación como «el acuerdo mediante el cual dos o más personas ponen en común, de una manera permanente, sus conocimientos o su actividad con un objetivo otro que el reparto de ganancias" (Parodi, 1993; 34). El reconocimiento por el Estado puede también adoptar la forma de exoneraciones fiscales en función de ciertos criterios, como por ejemplo en los Estados Unidos.

El reconocimiento por parte del Estado se manifiesta tambien en la utilización por los poderes públicos de un mismo término para designar un conjunto de organizaciones. Por ejemplo, el término “economía social» empezó a ser utilizado por varios gobiernos (francés, belga, canadiense y español, entre otros) para designar un gran conjunto de organizaciones que comprende las cooperativas, las mutualidades y las asociaciones sin fines de lucro que realizan actividades económicas. Por ejemplo en Francia, en 1981 el Estado creó la Delegación Interministerial para la Economia Social (Vienney, 1994), la Secretaría de Estado para la Economía Social, y en 1983, el Instituto de Desarrollo de la Economía Social (IDES), cuya misión es responder a las necesidades de financiamiento de las organizaciones pertenecientes a este sector.

El reconocimiento político de la economía social se ha ido extendiendo a otros paises europeos: en Bélgica, el gobierno regional de Wallonia creó un Consejo de la Economía Social, y en España se creó el Instituto Nacional de Promoción de la Economía Social. En cuanto a las instituciones de la Comunidad Europea, el Comité Económico y Social en todos sus trabajos utiliza la expresión «economía social" y la Comisión de las Comunidades Europeas creó una división de economia social, y recomienda la utilización de la expresión "economía social»" considerada como "la única, en el marco del Derecho y de las iniciativas políticas, que, a pesar de las imprecisiones y ambiguedades, permite ofrecer una imagen de este nuevo espacio estructurado en el seno del sistema económico" (Monzón y Barea, 1987; 27). La economía social integraría "todos los agentes que integren en una sola función objetivos económicos y objetivos sociales, y favorecen la participación de los ciudadanos y la corresponsabilidad en los procesos de toma de decisión” (Monzón y Barea, 1987; 25).

Finalmente, una tercera dimensión del reconocimiento es la hecha por el medio cientifico. Aqui, encontramos dos grandes definiciones del "tercer sector": una que sólo toma en cuenta las organizaciones sin fines de lucro de interés general (es el enfoque del John Hopkins Comparative Nonprofit Sector Project , y otra que incluye en una misma categoría 
las organizaciones de interés general y las organizaciones formadas en torno a los intereses de un grupo restringido de personas como las cooperativas y las mutualidades. Es la tendencia que predomina entre los investigadores franceses, belgas, españoles, canadienses, y en el CIRIEC. La revista de la sección canadiense del CIRIEC internacional cambió recientemente su nombre Coopératives et développementpor el de Economies et solidarites traduciendo de esta manera la ampliación de su orientación, desde las cooperativas hacia el conjunto de las organizaciones de la economía social. "Los actores de la economía social, que sean cooperativas, asociaciones o iniciativas de desarrollo local, se inscriben en una misma lógica original en lo que se refiere a las finalidades, la organización de una combinación adecuada de imperativos de solidaridad y de imperativos de mercado" (Presentación Economie et Solidarités, 1996; 1).

En muchos casos, ha sido a partir del esludio de las cooperativas que se ha desarrollado la investigación sobre las asociaciones. Estas tienen en común con las cooperativas el hecho de no ser formadas por inversionistas, como las empresas del sector privado, y de combinar una dimensión asociativa y una dimensión empresarial, económica. Las asociaciones, comparadas con los otros compo- nentes de la economía social, sobre todo las cooperativas, que han sido muy estudiadas, constituían una verdadera "terra incognita" que ha empezado a ser explorada sobre todo a partir de la década de 1980 , cuando se elabora un nuevo concepto para agrupar todas estas formas organizativas, que en el contexto de la crisis, conocen un proceso de multiplicación y diversificación ${ }^{1}$, y de reconocimiento mutuo.

Sin embargo, la delimitación y la categorización del campo de las asociaciones no dejan de plantear algunas dificultades y producir tensiones hasta el punto en que para algunos autores, "la idea de juntar todas las asociaciones bajo una misma nomenclatura participa de una estafa intelectual" porque no existe un criterio único que defina el grupo de las asociaciones. A diferencia de las cooperativas y las mutualidades, relativamente homogéneas, "no hay nada más heterogéneo que el universo de las asociaciones" (Kaminski, 1990, cit. por Malo, 1991; 44). Donde no existe un proceso de reconocimiento mutuo y una institucionalización del medio asociativo, es difícil definir rasgos que unifiquen el conjunto de las asociaciones. Se trata de un proceso que está todavía en su fase incipiente, en muchos paises.

Para varios autores, una diferencia fundamental existe entre las asociacio-

1 Al lado del movimiento cooperativo institucionalizado y de las organizaciones sin fines de lucro tradicionales, surgen nuevas modalidades asociativas como las empresas de inserción, las corporaciones de desarrollo económico comunitario, las nuevas cooperativas, las empresas sociales, los servicios de proximidad, etc. Sobre estas nuevas organizaciones existe una bibliografía abundante, ya que se trata de un campo de estudio en pieno auge en varios países. 
nes basadas en la auto-organización y las asociaciones basadas en la hetero-organización.

- La auto-organización designa la modalidad según la cual la asociación es un grupo voluntario de personas reciprocamente vinculadas por una relación de actividad y de asociación. Su identidad se define por la autoorganización puesto que se trata de grupos que se organizan para brindar servicios a sus miembros.

- La hetero-organización designa la modalidad según la cual la asociación se presenta bajo la forma de un grupo voluntario de personas vinculadas por una relación de actividad y una relación de asociación llevada a cabo en beneficio de otro grupo hacia el cual los miembros de la asociación se comprometen voluntariamente.

Se trata de una diferencia importante aúnque existen organizaciones que pertenecen a ambas catagorias ${ }^{2}$. (Gui, 1991) insiste en esta diferencia, que le parece fundamental. Plantea que toda organización microeconómica privada puede ser caracterizada por los dos criterios siguientes: el tipo de personas que controlan la actividad, es decir que poseen el último poder de decisión, y el tipo de personas beneficiarias, es decir a quienes está destinado de manera explicita o implícita el "excedente" de la organización. Sobre esta base, considera que la distinción más fundamental entre las diferentes formas posibles de organización se ubica entre las "organizaciones de interés mútuo" (mutual benefit organizations), en las cuales el grupo de personas que controla la actividad se confunde con el grupo de los beneficiarios, y las "organizaciones de interés general' ( $p u$ blic interest organizations), donde estos dos grupos no coinciden.

Sin embargo, existe un rasgo común entre estos dos tipos de organizaciones: el grupo de los beneficiarios no está constituído por inversionistas. Además, en ambos tipos de asociaciones debe realizarse un ensambiaje de dos dispositivos distintos y autónomos, la asociación de personas y la empresa (Marchal, 1990), y deben combinarse un objetivo social y de un objetivo económico.

Defourny, retomando la definición de la economía social propuesta por el Consejo Wallon de la Economía Social en 1990, y retomada en el Libro Blanco de la Economía Social en España (1992), señala que lo que constituye la especificidad fundamental de las asociaciones de la economía social es una ética particular que se traduce a nivel de las finalidades de la actividad y del modo de organización. «La economia social está formada por actividades económicas ejercidas por sociedades, particularmente cooperati-

Por ejemplo una asociación de consumidores defiende los intereses de sus miembros, pero su acción sirve al mismo tiempo a los intereses de los consumidores no miembros. También, historicamente, la multiplicación de asociaciones al servicio de sus miembros muchas veces desembocó en la creación de organizaciones de interés más general. 
vas, mutualidades y asociaciones cuya ética se traduce en los principios siguientes: 1) Finalidad de servicio a los miembros o a la colectividad en lugar de ganancia; 2) autonomía de gestión; 3) proceso de decisión democrático;4) primacía de las personas y del trabajo por sobre el capital en el reparto de los excedentes" (Defourny, 1995; 88). Defourny ve en esta ética la principal razón que explica la capacidad de las asociaciones para movilizar trabajo voluntario.

Otra dificultad en la delimitación de la economía social es la definición de la pertinencia económica de las asociaciones. Existe un acuerdo entre los cientificos para incluir en la economía social sólo las asociaciones con cierta pertinencia económica. Esto plantea la necesidad de definir una manera de evaluar la pertinencia económica.

Finalmente, otro problema que se plantea con la noción de economía social proviene de las diferencias que existen entre las diferentes unidades que forman el sector. Por ejemplo, numerosas cooperativas adoptan comportamientos $y$ estrategias que las diferencian cada vez menos de sus competidores del sector capitalista.

\section{Particularidades de la gestión asociativa}

Las asociaciones, como otras formas de organizaciones, toman decisiones y llevan a cabo actividades, con un medio que los obliga a recurrir a las técnicas de gestión. En el pasado, las instituciones de lo social eran instituciones de beneficiencia, que se financiaban me- diante donaciones y legados, viviendo de la caridad, fuese cristiana, laica u otra. Luego los poderes públicos empezaron a intervenir en el financiamiento de las asociaciones mediante subsidios y ayudas, a partir de la toma de conciencia por la colectividad de la utilidad pública de ciertas asociaciones. Pero, al igual que las donaciones, el subsidio era parte de una relación de asistencia. No preparaba a la gestión financiera activa. Bastaba para una organización tener un proyecto educativo, humanista o social. Muchas veces no se prestaba la debida atención a los costos de funcionamiento, con los consiguientes desperdicios y déficits.

Pero en años recientes, el financiamiento público mediante subsidios se ha venido transformando en un financiamiento contractual por objetivos. Mientras el subsidio era un monto otorgado unilateralmente, sin referencia precisa a la evaluación de un proyecto, el financiamiento mediante objetivos constituye otra manera de gerenciar los fondos públicos buscando una mayor eficiencia del dinero utilizado en los programas sociales, y una mayor responsabilidad frente al dinero. Generalmente, el financiamiento público sólo cubre una parte de los costos de funcionamiento, y las asociaciones mucho más que en el pasado deben recurrir al autofinanciamiento.

Entonces, las asociaciones han entrado a una etapa en la cual estan confrontadas al problema de la gestión económica. En este contexto, han conocido un conjunto de modificaciones: aumento del personal asalariado reclutado en base a criterios de profesionalismo; racionalización de la organización y de la gestión de los servi- 
cios; selección de las actividades en función de los objetivos y la misión pero tambien de las posibilidades del mercado.

- Estrategia de proyecto y estrategia de financiamiento

Según Jerome Boucler, se pueden distinguir en el enfoque estratégico de una asociación lo que está relacionado con su proyecto y lo que está relacionado con su financiamiento. Las asociaciones desarrollan estrategias de proyecto, que les dan su orientación y su legitimidad. Lo esencial de la estrategia de proyecto está contenido en el objeto de la asociación, y este objeto debe ser reactualizado constantemente, para asegurar la coherencia de los objetivos que deberían ser compartidos por el conjunto de los miembros. "Eje de identidad y de comunicación, el proyecto debe permitir clarificar los valores e integrarlos en una estrategia" (Boucler, 1994; 82).

Al lado de la estrategia de proyecto, existe un segundo tipo de estrategia que, al no ser asumida de manera adecuada, puede comprometer la primera; se trata de la estrategia de financiamiento.

El financiamiento mediante donaciones y subsidios públicos, que era la forma predominante del financiamiento de las asociaciones, no predisponía a una gestión eficiente. Además, otro factor influía en esta falta de integración de la gestión del dinero en las preocupaciones de los dirigentes de las asociaciones: se trata de la representación que se tenía de lo social y de lo económico como campos separados. Se veía lo social como algo que viene a reparar los daños causados por una economía dirigida por el dinero y la ganancia, de modo que lo social se consideraba como algo que no tenía mucho que ver con el dinero y la economía, aunque se necesitara el dinero para poder ejercer una función reparadora.

Hoy las asociaciones han operado un "viraje económico". Desde hace unos diez años, el mundo asociativo se encuentra en plena mutación, a consecuencia de la disminución del compromiso del Estado en materia de subvenciones. Muchas organizaciones han debido volverse prestatarias de servicios para seguir existiendo. Se trata de un fenómeno masivo y reciente, ligado a la crisis económica y a las crecientes necesidades sociales así como a la crisis del Estado de bienestar y a los procesos de descentralización y privatización. Para poder realizar sus objetivos en este contexto, las asociaciones deben "actuar de otra manera". Deben evitar acumular un déficit si quieren asegurar la viabilidad de su organización. Incluso, deben realizar un excedente para poder desarrollarla, emprender nuevas actividades, o evitar el abandono de ciertas actividades no rentables pero que son importantes en función de la misión de la asociación.

En este nuevo contexto, los cuadros directivos tienen una mejor formación, y tiende a imponerse en las asociaciones el modelo de la empresa, que se ha vuelto uel lugar ético central de la sociedad" (Rolin, 1995; 26). Los dirigentes asociativos son llevados a practicar la gestión científica, descubriendo las similitudes que existen entre sus organizaciones $y$ las empresas lucrativas. Hoy las grandes asociaciones sin fines de lucro 
tienen departamentos de marketing para difundir en la opinión pública los objetivos y las realizaciones, y asi captar donaciones y clientes.

Generalmente, las asociaciones sin fines de lucro utilizan diversas formas de financiamiento: contribuciones voluntarias de diferentes tipos: cotizaciones, donaciones de particulares en especies o en dinero, legados, mecenazgo de empresas, etc. Además, dentro de cada una de estas categorias, múltiples fórmulas son posibles. Asi por ejemplo, las donaciones en dinero de los particulares pueden ser directas en forma de pagos puntuales o por cuotas mediante colectas en la calle, a domicilio, etc. Pueden ser indirectas como en las «inversiones éticas" mediante las cuales el ahorrista acepta recibir un interés algo inferior a la tasa del mercado, cediendo así parte de la remuneración de su capital en beneficio de determinada asociación ${ }^{3}$. Se podría también considerar como donaciones financieras de los particulares las sumas intercambiadas contra bienes más o menos simbólicos cuyo precio de venta no tiene una relación directa con el costo de producción o con un precio de mercado: calcomanías, tarjetas de Amnistía Internacional, las tarjetas de Navidad de la Unicef, etc. Otro ejemplo de bien simbólico es la relación de padrinazgo con un niño de un país pobre que un organismo de ayuda internacional ofrece al público de los paises industrializados: por un peque- ño monto mensual, un donante puede apadrinar un niño africano, con el cual podrá comunicarse, intercambiar fotos, etc. En realidad el dinero no es entregado a cada niño, sino utilizado por la organización en diferentes programas tendientes a mejorar las condiciones de vida de la población. El solicitar ayuda financiera mediante el establecimiento de una relación de persona a persona con un niño constituye una estrategia de marketing que ha permitido motivar a la población de donantes, dándole un carácter menos abstacto a la solidaridad o a la caridad. Todas estas prácticas revelan un gran dinamismo $e$ ingeniosidad.

En respuesta a la crisis, desde los años ochenta asistimos a la multiplicación de nuevos tipos de asociaciones, donde se establece un vínculo diferente entre lo económico y lo social. Un ejemplo de este nuevo tipo de asociación lo constituyen las empresas de inserción, que han surgido como respuestas al problema del desempleo estructural, en particular de los jóvenes. Se trata de estructuras económicas - empresas que producen bienes y/o servicios para el mercado- pero con un objetivo social, la inserción social y profesional de poblaciones en dificultad, en particular de los jóvenes. En estas empresas se combina una actividad de formación profesional y también personal, y una actividad de producción, modificándose así el orden tradicional formación - trabajo. La actividad de formación

3 Por ejemplo, la Asociación de Circulos de Crédito de Montreal, la ACEM, capta los depósitos a plazo de individuos y colectividades (entre ellas varias comunidades religiosas), pagando a estos depositantes solidarios intereses un poco inferiores a los del mercado, y los fondos asi obtenidos son utilizados en pequenos créditos a iniciativas económicas populares. 
se financia en parte mediante la venta de los productos de la empresa, que debe ser rentable para que pueda lograrse el objetivo social. Otra parte de los recursos debe ser obtenida de la colectividad, y en particular del Estado, para cubrir los costos de la formación (pérdida de productividad debida a las características particulares de la fuerza de trabajo; necesidad de personal de apoyo a la actividad de formación: instructores, personal especializado dedicado a atender los problemas personales de los alumnos-trabajadores). El apoyo de la colectividad se puede tambien lograr a nivel de la compra de los productos. Por ejemplo, en una empresa de inserción canadiense, la producción de muebles está destinada a las guarderías y a las viviendas de los programas públicos de vivienda para familias de bajos ingresos, entre otros clientes. Para una relación calidad-precio igual, los organismos públicos (para los programas de vivienda) y comunitarios (en el caso de las guarderías), le otorgan la preferencia a la empresa de inserción, por solidaridad con su objetivo social.

Otra modalidad asociativa novedosa es la Corporación de Desarrollo Economico Comunitario (CDEC), donde se combinan varios actores sociales: organizaciones comunitarias, empresas privadas, sector público, para producir la revitalización de una zona deprimida. Este tipo de organización, que coordina intervenciones a nivel de la capacitación de la población y de la creación de empleo productivo, exige un salto cualitativo con respecto a las asociaciones del pasado: requiere de una apertura a nuevas estrategias, y de varios aprendizajes técnicos por la complejidad de las intervenciones a realizar ${ }^{4}$.

\section{- El equilibrio necesario entre las dos estrategias}

Siendo la asociación una combinación entre un objetivo social (razón de ser de la asociación de personas) y de una actividad económica, existe una doble amenaza para las asociaciones; la primera reside en perder de vista el proyecto en la gestión económica de las actividades; la segunda consiste en olvidar, en nombre de los objetivos sociales, las necesidades del equilibrio económico de las actividades y de las sanciones que resultan de este olvido (Boucler; 1994; 84).

La naturaleza económica de las asociaciones no las reduce a la dimensión de una empresa comercial. Son ante todo sociales antes de ser empresas. Si han desarrollado la dimensión empresarial, ha sido para cumplir con su misión social.

F. Rolin define tres grandes principios en los cuales se basa la ética asociativa. Son en primer lugar la primacia del hombre. Es la dimensión esencial de la asociación, que sea del tipo auto o hetero-organización.

El segundo principio es la capacidad de asociar. La asociación no es la propiedad de un círculo cerrado de miembros. A partir de este criterio de capaciEconómico Comunitario, Documento, Centro de Estudios de las Empresa, Facultad de Ciencias Econéomicas y Sociales, Universidad del Zulia, 1996. 
dad asociativa se puede cuestionar la pertenencia al sector de la economía social de un gran número de "asociaciones civiles" que son asociaciones sin miembros fuera de la junta directiva, o con una asamblea general que sólo funciona de una manera formal, quedando todo el poder en manos de los directivos ${ }^{5}$.

El tercer principio es la no lucratividad. En algunas legislaciones, se establece esta condición en el texto mismo de ley. Esta característica constituye la diferencia esencial entre una asociación y una empresa comercial. Los miembros de una asociación no pueden ser asimilados a los socios de una empresa comercial que aportan un capital y esperan un rendimiento financiero. La legitimidad social de la asociación se basa en el interés colectivo o general que defiende, asi como su capacidad para movilizar diferentes tipos de aportes voluntarios, en dinero y en tiempo de trabajo.

\section{- El desarrollo de un nuevo em- presariado social}

Entre los actores de las asociaciones de la economia social se puede distinguir un grupo cuya importancia es central: los promotores, los que actúan como portadores de los proyectos, que solicitan la participación de los asociados y organizan el proceso de creación colectiva. Estos actores promotores de la asociación se vuelven los empresarios de la actividad económica desarrollada por la asociación. Deben ser capaces de captar el impulso asociativo, dominar estrategias sofisticadas en sus relaciones con las instituciones privadas y públicas, coordinar y gerenciar muchos tipos de recursos. Según Laville $(1991 ; 104)$, se trata de una nueva figura de empresariado social cuyas competencias difieren considerablemente de las exigidas para el creador de empresas tradicionales. Deben haber desarrollado competencias en la gestión de proyectos, y también en la animación de redes y en la mediación política. Haber constituído recursos en los tres campos en que se apoyan para el desarrollo de la empresa asociativa: el campo público (relaciones con los diferentes niveles de gobierno), el campo privado (para la gestión de un proyecto económico y la obtención de la participación del sector privado ${ }^{6}$ ).

Diferente del empresario clásico, el empresario social lo es también del actor de masas en que no impulsa el cambio mediante el conflicto colectivo sino mediante microacciones colectivas, soportes de interacciones duraderas que resultan

5 Es el caso de numerosas asociaciones civiles en Venezuela, sobre todo de las ONG que se crearon para ejecutar los nuevos programas sociales del Estado (Programa de Multihogares, Programa de Formación y Capacitación Juvenil, etc). Varias de estas asociaciones dificilmente podrían incluirse en el sector de la economia social, tal como ha sido definido en la Carta de la Economia Social (ver arriba), porque son ante todo organizaciones privadas con fines de lucro, en particular las asociaciones que administran veinte o más multihogares.

La participación del sector privado puede darse bajo la forma de donaciones, de compra de los bienes y/o servicios producidos por la organización, y en la forma de participación en el Consejo de Administración de la asociación, aportando en este caso la experticia desarrollada en el campo de la empresa privada lucrativa. 
de una identidad y voluntad comunes. Se da por tarea abrir sistemas bloqueados constituyendo espacios propios, en un contexto de crisis en que se agotan las posibilidades de promoción social y se desplazan las fronteras institucionales antes estabilizadas en un contexto de expansión.

Estos empresarios sociales son interactores que agrupan en torno a su proyecto actores asociados que son de diversos tipos (Laville, 1991; 106):

- asociados técnicos, expertos o profesionales que son movilizados en función de su saber o saber-hacer en campos particulares;

- asociados notables, que son movilizados en función de los lugares que ocupan en las redes institucionales, para que puedan contribuir en drenar recursos hacia la asociación, contrarrestando los modos de gestión compartimentados de la esfera pública y los corporatismos de la esfera priva$\mathrm{da}_{\mathrm{i}}$

- asociados militantes, movilizados en función de su pertenencia a redes que pueden poner a disposición del proyecto recursos no monetarios;

- asociados beneficiarios o usuarios, que aportan su participación en la definición de modos de acción adaptados a las necesidades sociales, en la difusión de la utilidad social del proyecto y de la asociación y en presión hacia las autoridades políticas para el reconomiento público de las actividades realizadas.
Estos diferentes tipos de asociados se diferencian de las formas de representación de intereses presentes en las instancias de negociación colectiva. Los asociados representan aqui las instituciones a las que pertenecen sobre la base de un compromiso personal, y no de un mandato. Se movilizan a partir de valores compartidos con otros actores - por ejemplo la creencia en la necesidad de una inserción social y profesional de los jóvenes en un contexto de creciente deserción escolar y exclusión social de las jóvenes generaciones-, Sin identidad común al inicio, los miembros de la asociación dan como objeto de su reflexión la elaboración de servicios sociales tomando en cuenta la crisis de la sinergía Estado-mercado ${ }^{7}$. A partir de una referencia común a valores afirmados en un proyecto y validados por la adhesión voluntaria de los miembros, se encuentran reunidas las condiciones portadoras de las innovaciones. Se vuelve posible abandonar el mundo de relaciones en el cual cada uno se sitúa exclusivamente en relación a su institución para acceder a lo que JeanLouis Laville (1991) llama zun régimen de transversalidad" en el cual cada uno puede expresar no solamente lo que implica su estatus sino también lo que es como persona sensibilizada ante tal o cual problema, expresando así una posición subjetiva, transcendiendo sus roles sociales.

7 La explicación de este punto está desarrollada en M. Richer, «Economia y solidaridad. Esbozo de una problemática * Cuadernos del Programa de Gerencia Comunitaria, Centro de Estudios de la Empresa, FACES, LUZ, 1996. 


\section{Conclusión}

En el contexto de la crisis, que es a la vez una crisis de la economia, que se origina en el desarrollo tecnológico y la mundialización de la economía, y una crisis del Estado, cuyo déficit ya no le permite absorber los costos sociales del modelo de desarollo, se plantean nuevos desafíos a las asociaciones. Estas, si bién han existido desde siempre, habian perdido algo de su actualidad durante el periódo de auge del Estado de bienestar (o, en América latina, del modelo populista). La nueva importancia que va adquiriendo el "tercer sector" sobre todo a partir de la década del $80^{\prime}$ ha llevado a un creciente interés de las ciencias sociales, y entre ellas las ciencias de la gestión, por este tipo de organizaciones.

Algunos investigadores se han dedicado más a las organizaciones sin fines de lucro, llamadas en el mundo anglosajon Nonprofit sector, otros, sobre todo en los países francofonos, pero también en España, han empezado a reactualizar el concepto de economía social, que engloba las cooperativas, las sociedades mutualistas $y$ las asociaciones $\sin$ fines de lucro que realizan alguna actividad económica.

La gestión de las empresas de la economía social es compleja: debe abarcar las dos dimensiones que tienen estas organizaciones, la dimensión asociación y la dimensión empresa. Debe mobilizar personas en torno a una misión social, $y$ al mismo tiempo obtener los recursos necesarios a la realización de esta misión mediante una combinación de estralegias de financiamiento: donaciones, produc- ción de bienes y/o servicios para el mercado, obtención de financiamiento público. El desarrollo de la competencia entre las organizaciones por la captación de las donaciones, asi como el nuevo comportamiento del sector público (financiamiento otorgado mediante convenios en torno a proyectos en lugar de subsidios indiscriminados), obliga a las asociaciones a desarrollar una mayor capacidad empresarial en el arranque económico de los proyectos, en la búsqueda de mercados, en la búsqueda de fondos, en la negociación de un acceso a los mercados púbiicos, en la búsqueda de asociados para el desarrollo de los proyectos, en la formación de redes entre asociaciones, etc.

Varios autores ven en el desarrollo de la economía social una importante contribución al desarrollo social. Las ciencias de la gestión, y las escuelas de administración, deben interesarse más por este objeto, y asi contribuir a desarrollar una sinergía entre el mundo académico y el mundo de las asociaciones.

\section{Referencias Bibliográficas}

Alfandari, E. (1984) "L'économie sociale à la recherche d'une définition*, Revue de l'économie sociale, No. 1, julio-septiembre.

Anheier, H.K. y W. Seibel (eds.) (1990). The Third Sector Comparative Studies of Nonprofit Organizations, de Gruyter, New York.

Boucler, J. (1994). "Management associatif et stratégie», Revue internationales de l'économie sociale, 255, P. 81-89.

Defourny, J. (1995) «Apports et limites de trois approches courantes des associations", Coopératives et développement, 26, 1, p. 81-101. 
Defourny, J. (1992), "Histoire et actualité du fait associatifm, CIRIEC Working Papers No. 10.

Gui, B. (1991). "The Economic Rationale for the Third Sectorn, Annals of Public and Cooperative Economics, 62, 4, p. 551-572.

Hansmann, H. (1987), "Economic Theories of Nonprofit Organization», Powell, W.W.ed,. The Nonprofit Sector, Yale University Press, New Haven.

Kendall, J., et al. (1992). "La economía social en el Reino Unido*, en Monzon y Defourny (1992), p. 89-130.

Klein, J.-L. y B. Lévesque (1995). Contre l'exclusion, repenser l'économle, Presses de P'Université du Québec, Québec.

Kramer, R.M. (1990), "Nonprotit Social Service Agencies and the Welfare State: Some Research Considerations", en Anheler, H.K. y W. Seibel.

Laville, J.L. (1991) eles nouveaux services de proximité prolongementos et ruptures par rapport à l'économie sociale", Revue des études coopératives, mutuallstes et associatives, 39 , 3er trimestre 1991.

Malo, M.-C. (1991). "Les associacions au sein de l'économie sociale", Inter-Action, Les Editions du CEPAQ, Montréal.

Marchal, E. (1990). Du désintéressement au marché: les différentes formes d'entreprises associatives. Centre $d^{\prime}$.
Études de l'Emploi, Dossier de recherche No. 33, París.

Monzón, J.L.y J. Defourny, dir. (1992), Economia soclal. Entre Economía Capitalista y Economía Pública, CIRIEC. España.

Monzón, J.L. y J. Barea Tejeiro (1987). Le Livre Blanc de l'économie sociale en Espagne, CIRIEC-Espagne.

Parodi, M. (1995) "La riche nature ou la double ambivalence des associations du secteur sanitaire et socialn, Revue Internationale de l'économie sociale, 260, p. $32-41$.

Richer, Madeleine (1996) "Economia y solidaridad. Esbozo de una problemática". Cuadernos del Programa de Gerencia Comunitaria. Centro de Estudios de la Empresa. Universidad del Zulia. Maracaibo-Venezuela.

Rock, Ch. y M. Klinedinst (1992). "El sector de "economía social" en los Estados Unidos", en Monzon y Defourny, ob. cit.

Rolin, F. (1995), "Quand l'argent rentre en compten, Economie et Humanisme, 332, marzo, p. 23-28).

Vienney, C. (1994) L'économie sociale, La Découverte, París.

Weisbrod, B. (1988). The Nonprofit Economy, Harvard University Press, Cambridge, Mass. 